International Mathematical Forum, Vol. 9, 2014, no. 27, 1333 - 1340

HIKARI Ltd, www.m-hikari.com

http://dx.doi.org/10.12988/imf.2014.47565

\title{
Numerical Solution of Interval Linear Programming
}

\author{
Huiling Duan and Tao Peng \\ School of Mathematics and Statistics \\ Chongqing Three Gorges University \\ Chongqing, 404100, P. R. China
}

Copyright (c) 2014 Huiling Duan and Tao Peng. This is an open access article distributed under the Creative Commons Attribution License, which permits unrestricted use, distribution, and reproduction in any medium, provided the original work is properly cited.

\begin{abstract}
This paper presents an algorithm for solving interval linear programming(ILP) problems. Interval inequality constraints and equality constraints are discussed separately. The aim of the paper is to show that (ILP) problems can be decomposed into two general linear programming(LP) by the monotonicity of (LP) problems, and we can gain the interval objective values. Finally, the proposed method have virtually the same results with paper [1].
\end{abstract}

Keywords: interval linear programming, interval numbers, optimal objective interval values

\section{Introduction}

In traditional mathematical programming, the coefficients of the problems are always treated as deterministic values. However, uncertainty always exits in practical engineering problems[1-3]. In order to deal with the uncertain optimization problems, fuzzy[4] and stochastic[5-6] approaches are frequently used to describe the imprecise characteristics. In these two approaches, the membership function and probability distribution play important roles. However, it is sometimes difficult to specify an appropriate membership function or accurate probability distribution in an uncertain environment[7]. Therefore, interval optimization problems may provide an alternative choice for solving uncertainty optimization problems. 
The solution methods to interval linear programming(ILP) problems were explored by some scholars [1][7-9]. An interval linear programming is defined as follows:

$$
\begin{aligned}
& \min z=C^{I} X \\
& \text { s.t. } \quad A^{I} X \leq b^{I}, \\
& \quad x_{j} \geq l_{j}, j=1, \ldots, n .
\end{aligned}
$$

Where $C^{I} \in\left\{R^{ \pm}\right\}^{1 \times n}, b^{I} \in\left\{R^{ \pm}\right\}^{n \times 1}, A^{I} \in\left\{R^{ \pm}\right\}^{m \times n}, R^{ \pm}$denotes a set of interval numbers, and $X=\left(x_{1}, \cdots, x_{n}\right)^{T}$ is an n-dimensional design vector.

\section{A general model}

In this paper, a more general model of interval linear programming (ILP) than (1) is defined as follows:

$$
\begin{array}{ll}
\min & z=\sum_{j=1}^{n} c_{j}^{I} x_{j} \\
\text { s.t. } & \sum_{j=1}^{n} a_{i j}^{I} x_{j} \leq b_{i}^{I}, i=1, \ldots, l, \\
& \sum_{j=1}^{n} a_{i j}^{I} x_{j}=b_{i}^{I}, i=l+1, \ldots, m, \\
& x_{j} \geq l_{j}, j=1, \ldots, n .
\end{array}
$$

where $c_{j}^{I} \in\left[c_{j}^{L}, c_{j}^{U}\right], a_{i j}^{I} \in\left[a_{i j}^{L}, a_{i j}^{U}\right], b_{i}^{I} \in\left[b_{i}^{L}, b_{i}^{U}\right], 0<l_{j}^{I} \in\left[l_{j}^{L}, l_{j}^{U}\right]$ are interval numbers.

Let $S=\left\{\left(c_{j}^{I}, a_{i j}^{I}, b_{i}^{I}, l_{j}^{I}\right) \mid c_{j}^{I} \in\left[c_{j}^{L}, c_{j}^{U}\right], a_{i j}^{I} \in\left[a_{i j}^{L}, a_{i j}^{U}\right], b_{i}^{I} \in\left[b_{i}^{L}, b_{i}^{U}\right], l_{j}^{I} \in\right.$ $\left.\left[l_{j}^{L}, l_{j}^{U}\right], i=1, \ldots, m, j=1, \ldots, n\right\}$.

About programming (2), there are some discussions as follows. This paper's innovation lies in theorem 3.3, by which we can decompose programming (2) into two deterministic linear programming to gain the optimal objective interval values of the interval linear programming (2).

\section{Results and Discussion}

\subsection{Equality constraints}

Theorem 3.1. In programming (2), denote $A=\left\{X \mid \sum_{j=1}^{n} a_{i j}^{I} x_{j}=b_{i}^{I}, \forall a_{i j}^{I} \in\right.$ $\left.\left[a_{i j}^{L}, a_{i j}^{U}\right], x_{j} \geq l_{j}^{I}, b_{i}^{I} \in\left[b_{i}^{L}, b_{i}^{U}\right], l_{j}^{I} \in\left[l_{j}^{L}, l_{j}^{U}\right], i=l+1, \ldots, m, j=1, \ldots, n\right\}, B=$ $\left\{X \mid \sum_{j=1}^{n} a_{i j}^{L} x_{j} \leq b_{i}^{U}, \sum_{j=1}^{n} a_{i j}^{U} x_{j} \geq b_{i}^{L}, x_{j} \geq l_{j}^{I}, l_{j}^{I} \in\left[l_{j}^{L}, l_{j}^{U}\right], i=l+1, \ldots, m, j=\right.$ $1, \ldots, n\}$, then $A=B$. 
Proof. Firstly, proofing $A \subseteq B . \forall X^{*} \in A, \exists a_{i j}^{I} \in\left[a_{i j}^{L}, a_{i j}^{U}\right], b_{i}^{I} \in\left[b_{i}^{L}, b_{i}^{U}\right], l_{j}^{I} \in$ $\left[l_{j}^{L}, l_{j}^{U}\right]$, s.t. $\sum_{j=1}^{n} a_{i j}^{I} x_{j}^{*}=b_{i}^{I}, x_{j}^{*} \geq l_{j}^{I}(\geq 0)$. Then $\sum_{j=1}^{n} a_{i j}^{L} x_{j}^{*} \leq \sum_{j=1}^{n} a_{i j}^{I} x_{j}^{*}=b_{i}^{I} \leq b_{i}^{U}$ and $\sum_{j=1}^{n} a_{i j}^{U} x_{j}^{*} \geq \sum_{j=1}^{n} a_{i j}^{I} x_{j}^{*}=b_{i}^{I} \geq b_{i}^{L}$. Clearly, $X^{*} \in B . A \subseteq B$ holds.

Secondly proofing $B \subseteq A . \forall X^{*} \in B$, assuming the function $f\left(a_{i 1}^{I}, a_{i 2}^{I}, a_{i 3}^{I}, \ldots, a_{i n}^{I}\right)=$ $\sum_{j=1}^{n} a_{i j}^{I} x_{j}^{*}(i=l+1, \ldots, m)$ are continuous on $\left[a_{i 1}^{L}, a_{i 1}^{U}\right] \times\left[a_{i 2}^{L}, a_{i 2}^{U}\right] \times \ldots \times\left[a_{i n}^{L}, a_{i n}^{U}\right]$, because $\forall X^{*} \in B$, we have $\sum_{j=1}^{n} a_{i j}^{L} x_{j}^{*} \leq b_{i}^{U}$ and $\sum_{j=1}^{n} a_{i j}^{U} x_{j}^{*} \geq b_{i}^{L}$. And then we give a $a_{i j}^{I} \in\left[a_{i j}^{L}, a_{i j}^{U}\right]$ in order to $b_{i}^{L} \leq \sum_{j=1}^{n} a_{i j}^{I} x_{j}^{*} \leq b_{i}^{U}$. Since the intermediate value theorem, $\exists b_{i}^{I} \in\left[b_{i}^{L}, b_{i}^{U}\right]$, s.t. $\sum_{j=1}^{n} a_{i j}^{I} x_{j}^{*}=b_{i}^{I}$, and so $X^{*} \in A$. Therefore, $B \subseteq A$ holds. From above, we can attain $A=B$.

From theorem 3.1, we know that $\sum_{j=1}^{n} a_{i j}^{I} x_{j}=b_{i}^{I}$ is equivalent to $\sum_{j=1}^{n} a_{i j}^{L} x_{j} \leq$ $b_{i}^{U}$ and $\sum_{j=1}^{n} a_{i j}^{U} x_{j} \geq b_{i}^{L}$. So the minimum optimal solution of interval programming (2) must satisfy both $\sum_{j=1}^{n} a_{i j}^{L} x_{j} \leq b_{i}^{U}$ and $\sum_{j=1}^{n} a_{i j}^{U} x_{j} \geq b_{i}^{L}$.

Lemma 3.2 (see[1]). The maximum optimal solution of interval programming (2) must satisfy one of $\sum_{j=1}^{n} a_{i j}^{L} x_{j}=b_{i}^{U}$ and $\sum_{j=1}^{n} a_{i j}^{U} x_{j}=b_{i}^{L}$.

\subsection{The monotonicity of programming (3.1)}

Assuming $c_{j}, a_{i j}, b_{i}, l_{j}(i=1, \cdots, j)$ are real numbers of $\left[c_{j}^{L}, c_{j}^{U}\right],\left[a_{i j}^{L}, a_{i j}^{U}\right],\left[b_{i}^{L}, b_{i}^{U}\right]$, $\left[l_{j}^{L}, l_{j}^{U}\right]$ respectively, programming (2) will be a general linear programming(LP) problems:

$$
\begin{aligned}
& \min z=\sum_{j=1}^{n} c_{j} x_{j} \\
& \text { s.t. } \quad \sum_{j=1}^{n} a_{i j} x_{j} \leq b_{i}, i=1, \ldots, l, \\
& \quad x_{j} \geq l_{j}, j=1, \ldots, n .
\end{aligned}
$$

where has no equation constraints.

Suppose that $S^{\prime}$ is the feasible region of programming $(2), X_{S^{\prime}}^{*}$ is the optimal solution of programming $(3)$, and $G=\min _{X \in S^{\prime}} z(X)=z\left(X_{S^{\prime}}^{*}\right)$.

Clearly, if $c_{j}, a_{i j}, b_{i}, l_{j}(i=1, \cdots, l, j=1, \cdots, n)$ are deterministic values of $\left[c_{j}^{L}, c_{j}^{U}\right],\left[a_{i j}^{L}, a_{i j}^{U}\right],\left[b_{i}^{L}, b_{i}^{U}\right],\left[l_{j}^{L}, l_{j}^{U}\right]$ respectively, $G=G\left(c_{j}, a_{i j}, b_{i}, l_{j}\right)$ is 
the function of $c_{j}, a_{i j}, b_{i}, l_{j}$. Now, we will explain the relation between $G=$ $G\left(c_{j}, a_{i j}, b_{i}, l_{j}\right)$ and $c_{j}, a_{i j}, b_{i}, l_{j}$, which is an important characteristic of programming (3).

Theorem 3.3. $G$ is an increasing function of $c_{j}, a_{i j}, l_{j}$ and decreasing function of $b_{i}$, namely, $\frac{\partial G}{\partial c_{j}} \geq 0, \frac{\partial G}{\partial a_{i j}} \geq 0, \frac{\partial G}{\partial l_{j}} \geq 0, \frac{\partial G}{\partial b_{i}} \leq 0$.

Proof. For $c_{j}$, giving an increasing variable $\Delta c_{j}>0, c_{j}+\Delta c_{j}>0$, then we can get $0 \leq \sum_{i=1}^{n} c_{j} x_{j} \leq \sum_{i=1}^{n}\left(c_{j}+\Delta c_{j}\right) x_{j}$, namely, $G\left(c_{j}, a_{i j}, b_{i}, l_{j}\right) \leq G\left(c_{j}+\right.$ $\left.\Delta c_{j}, a_{i j}, b_{i}, l_{j}\right)$, therefore $\frac{\partial G}{\partial c_{j}} \geq 0$.

For $a_{i j}$, giving an increasing variable $\Delta a_{i j}>0, a_{i j}+\Delta a_{i j}>0$, let $S_{1}=$ $\left\{X \mid \sum_{j=1}^{n} a_{i j} x_{j} \leq b_{i}, i=1, \ldots, l\right\}, S_{2}=\left\{X \mid \sum_{i=1}^{n}\left(a_{i j}+\Delta a_{i j}\right) x_{j} \leq b_{i}, i=1, \ldots, l\right\}$. If $X \in S_{2}, \sum_{i=1}^{n}\left(a_{i j}+\Delta a_{i j}\right) x_{j} \leq b_{i}, i=1, \ldots, l$. However, $\sum_{j=1}^{n} a_{i j} x_{j} \leq \sum_{j=1}^{n}\left(a_{i j}+\Delta a_{i j}\right) x_{j}$ $\leq b_{i}, i=1, \ldots, l$, therefore, $X \in S_{1}$. And then $S_{2} \subseteq S_{1}$, let $S_{1}=S_{2} \cup T$, $S_{2} \cap T=\varphi$, so $\left.G\right|_{X \in S_{1}}=\min \left\{\left.G\right|_{X \in S_{2}},\left.G\right|_{X \in T}\right\} \leq\left. G\right|_{X \in S_{2}}$, namely, $\frac{\partial G}{\partial a_{i j}} \geq 0$.

Similarly, we can have $\frac{\partial G}{\partial l_{j}} \geq 0, \frac{\partial G}{\partial b_{i}} \leq 0$.

We only discuss inequality constraints $(i=1, \cdots, l, j=1, \cdots, n)$ in programming (3), for equality constraints $\sum_{j=1}^{n} a_{i j}^{I} x_{j}=b_{i}^{I}$ equivalent to $\sum_{j=1}^{n} a_{i j}^{L} x_{j} \leq$ $b_{i}^{U}$ and $\sum_{j=1}^{n} a_{i j}^{U} x_{j} \geq b_{i}^{L}$ or one of $\sum_{j=1}^{n} a_{i j}^{L} x_{j}=b_{i}^{U}$ and $\sum_{j=1}^{n} a_{i j}^{U} x_{j}=b_{i}^{L}$, which are all deterministic inequality constraints, and won't affect programming (2)'s monotonicity .

\subsection{The lower and upper bound of programming (2)}

As in [1], in order to compute the interval numbers of objective function , we need to find the lower and upper bound of the objective function value of programming (2).

To calculate the bounds of programming (2) by theorem (3.1), lemma(3.2) and theorem(3.3), programming (2) can be translated into the following two programming problems (4) and (5). 


$$
\begin{array}{cl}
z^{L}= & \min _{\left(c_{j}^{I}, a_{i j}^{I}, b_{i}^{I}, l_{j}^{I}\right) \in S} \min _{X} z=\sum_{j=1}^{n} c_{j}^{I} x_{j} \\
\text { s.t. } & \sum_{j=1}^{n} a_{i j}^{I} x_{j} \leq b_{i}^{I}, i=1, \ldots, l, \\
& \sum_{j=1}^{n} a_{i j}^{L} x_{j} \leq b_{i}^{U}, i=l+1, \ldots, m, \\
& \sum_{j=1}^{n} a_{i j}^{U} x_{j} \geq b_{i}^{L}, i=l+1, \ldots, m, \\
& x_{j} \geq l_{j}, j=1, \ldots, n . \\
z^{U}= & \max _{\left(c_{j}^{I}, a_{i j}^{I}, b_{i}^{I}, l_{j}^{I}\right) \in S} \min _{X} z=\sum_{j=1}^{n} c_{j}^{I} x_{j} \\
\text { s.t. } \quad \sum_{j=1}^{n} a_{i j}^{I} x_{j} \leq b_{i}^{I}, i=1, \ldots, l, \\
\quad \sum_{j=1}^{n} a_{i j}^{L} x_{j}=b_{i}^{U}, i=l+1, \ldots, m, \\
\text { (or } \left.\quad \sum_{j=1}^{n} a_{i j}^{U} x_{j}=b_{i}^{L}, i=l+1, \ldots, m,\right) \\
\quad x_{j} \geq l_{j}, j=1, \ldots, n .
\end{array}
$$

In programming (4) and (5), programming (2)'s equality constrains have been translated into corresponding inequality constraints.

For the objective function in (4), $z^{L}$ can be written as $z^{L}=\min _{\left(c_{j}, a_{i j}, b_{i}, l_{j}\right) \in S} \min _{X \in S^{\prime}} z=$ $\sum_{j=1}^{n} c_{j}^{I} x_{j}$, according to theorem $3.3, G$ is an increasing function of $c_{j}$, so $z^{L}=\min _{\left(c_{j}, a_{i j}, b_{i}, l_{j}\right) \in S} G\left(c_{j}, a_{i j}, b_{i}, l_{j}\right)=\min _{X} z=\sum_{j=1}^{n} c_{j}^{L} x_{j}$, which is as same as the result of [1].

For the inequality constraints in (4) $\sum_{j=1}^{n} a_{i j}^{I} x_{j} \leq b_{i}^{I}, i=1, \ldots, l$, and $x_{j} \geq$ $l_{j}^{I}, j=1, \ldots, n$, according to theorem $3.3, G$ is the increasing function of $a_{i j}, l_{j}$ and is the decreasing function of $b_{i}$, so that in order to calculate the lower bound, $\sum_{j=1}^{n} a_{i j}^{I} x_{j} \leq b_{i}^{I}, i=1, \ldots, l, x_{j} \geq l_{j}^{I}, j=1, \ldots, n$ can be written as $\sum_{j=1}^{n} a_{i j}^{L} x_{j} \leq b_{i}^{U}, i=1, \ldots, l$ and $x_{j} \geq l_{j}^{L}, j=1, \ldots, n$.

Similarly, we write $z^{U}$ of $(5)$ as $z^{U}=\max _{\left(c_{j}, a_{i j}, b_{i}, l_{j}\right) \in S} G\left(c_{j}, a_{i j}, b_{i}, l_{j}\right)=\min _{X} z=\sum_{j=1}^{n} c_{j}^{U} x_{j}$, and inequality constraints $\sum_{j=1}^{n} a_{i j}^{I} x_{j} \leq b_{i}^{I}, i=1, \ldots, l$, and $x_{j} \geq l_{j}^{I}, j=1, \ldots, n$ can be written as $\sum_{j=1}^{n} a_{i j}^{U} x_{j} \leq b_{i}^{L}, i=1, \ldots, l$ and $x_{j} \geq l_{j}^{U}, j=1, \ldots, n$. 
As a consequence, the smallest and largest objective value for $\mathrm{z}$ can be determined by mathematical programming problems

$$
\begin{array}{ll}
z^{L}= & \min _{X} \sum_{j=1}^{n} c_{j}^{L} x_{j} \\
\text { s.t. } & \sum_{j=1}^{n} a_{i j}^{L} x_{j} \leq b_{i}^{U}, i=1, \ldots, l \\
& \sum_{j=1}^{n} a_{i j}^{L} x_{j} \leq b_{i}^{U}, i=l+1, \ldots, m \\
& \sum_{j=1}^{n} a_{i j}^{U} x_{j} \geq b_{i}^{L}, i=l+1, \ldots, m \\
& x_{j} \geq l_{j}, j=1, \ldots, n
\end{array}
$$

and

$$
\begin{array}{ll}
z^{U}= & \min _{X} \sum_{j=1}^{n} c_{j}^{U} x_{j} \\
\text { s.t. } & \sum_{j=1}^{n} a_{i j}^{U} x_{j} \leq b_{i}^{L}, i=1, \ldots, l \\
& \sum_{j=1}^{n} a_{i j}^{U} x_{j}=b_{i}^{L}, i=l+1, \ldots, m \\
\left(\text { or } \quad \quad \sum_{j=1}^{n} a_{i j}^{L} x_{j}=b_{i}^{U}, i=l+1, \ldots, m\right) \\
\quad x_{j} \geq l_{j}, j=1, \ldots, n
\end{array}
$$

Both programming (6) and (7) are traditional linear programming, and we can easily obtain the global optimum solution and the associated objective values $z^{L}, z^{U}$, the lower and upper bound of the objective values of the interval linear programming (2). We can find programming (6) and (7) is the same as [1]'s models. So the result is coincident. We will illustrate this fact further by an example in the next section.

\subsection{Example}

Example (see[1])

$$
\begin{array}{ll}
\min & z=[-1,2] x_{1}+x_{2} \\
\text { s.t. } & -x_{1}+[1,2] x_{2} \geq[-2,-1], \\
& {[2,3] x_{1}+x_{2}=[3,4]} \\
& x_{2} \leq 3 \\
& x_{1}, x_{2} \geq 0 .
\end{array}
$$


According to analysis above, we can obtain

$$
\begin{array}{ll}
z^{L}= & \min z=-x_{1}+x_{2} \\
\text { s.t. } & x_{1}-2 x_{2} \leq 2 \\
& 2 x_{1}+x_{2} \leq 4 \\
& 3 x_{1}+x_{2} \geq 3 \\
& x_{2} \leq 3 \\
& x_{1}, x_{2} \geq 0 .
\end{array}
$$

and

$$
\begin{gathered}
z^{U}=\min z=2 x_{1}+x_{2} \\
\text { s.t. } \quad x_{1}-x_{2} \leq 1, \\
2 x_{1}+x_{2}=4 \\
\text { or } \left.3 x_{1}+x_{2}=3\right) \\
x_{2} \leq 3 \\
x_{1}, x_{2} \geq 0 .
\end{gathered}
$$

we can obtain the programming of $z^{L}, z^{U}$. By using the function "linprog" in Matlab 7.0, we can calculate $z^{L}, z^{U}$ of two examples, whose results are listed in Table 1.

\begin{tabular}{|c|c|}
\hline & Example 1 \\
\hline This Paper's result & $z^{L}=-2, x_{1}=2, x_{2}=0, z^{U}=4, x_{1}=1.2377, x_{2}=1.5246$ \\
\hline [1]'s result & $z^{L}=-2, x_{1}=2, x_{2}=0, z^{U}=4, x_{1}=5 / 3, x_{2}=2 / 3$ \\
\hline
\end{tabular}

Table 1: numerical examples' results

In Table 1, this paper's result (lower bond $z^{L}$, and upper bound $z^{U}$ ), $\left[z^{L}, z^{U}\right]=[-2,2]$, is the same as the [1], which can illustrate our method well.

\section{Conclusion}

In order to find optimal objective interval values $\left[z^{L}, z^{U}\right]$, Guo[1] discussed (ILP)'s objective function, inequality constraints and equality constraints separately. However, in this paper we needn't discuss these with hard work but equality constraints, and for monotonicity of function $\mathrm{G}$, we can easily gain the same result.

The method deserves to be considered widely while solving other interval programming problems, such as interval nonlinear programming. 


\section{References}

[1] J. P. Guo, W.H.Li, Standard form of interval linear programming and its optimal objective interval value, Journal of Management Sciences in China, 3 (2004), 59 - 63.

[2] W. L, X. Tian, Numerical solution method for general interval quadratic programming, Applied Mathematics and Computation, 202(2008), 589 595 .

[3] S.T. Liu, R.T. Wang. A numerical solution method to interval quadratic programming, Applied Mathematics and Computation, 189(2007), 1274 1281.

[4] C. Jiang, X. Han, F.J. Guan, Y.H. Li. An uncertain structural optimization method based on nonlinear interval number programming and interval analysis method, Engineering Structures, 29(2007), 3168-3177.

[5] B.D.Liu, Iwamura, K.. Fuzzy programming with fuzzy decisions and fuzzy simulation-based genetic algorithm, Fuzzy Sets and Systems, 2001, 122,PP:253-262.

[6] Cho, Gyeong-Mi. Log-barrier method for two-stage quadratic stochastic programming, Applied Mathematics and Computation, 164(2005), 45-69.

[7] B.D.Liu, R.Q.Zhao, G.Wang. Uncertain Programming with Applications, Tsinghua University Press, Beijing, China, 2003.

[8] A. Sengupta,T.K. Pal, D. Chakraborty. Interpretation of inequality constraints involving interval coefficients and a solution to interval linear programming, Fuzzy Sets and Systems, 119(2001), 129-138.

[9] P. Serafini. Linear programming with variable matrix entries, Operations Research Letters ,33(2005), 165 - 170.

[10] W.Li, G.X.Wang. General solutions for linear programming with interval right hand side, in: Xizhao Wang, Daniel Yeung, Xiaolong Wang (Eds.), Proceedings of ICMLC, Dalian, china, 3(2006), 1836 - 1839.

Received: July 11, 2014 